\title{
Approaching the quantum limit of precision in absorbance estimation using classical resources
}

\author{
Euan J. Allen $\odot,{ }^{1,2, *}$ Javier Sabines-Chesterking, ${ }^{1}$ Alex R. McMillan, ${ }^{1}$ Siddarth K. Joshi $\odot,{ }^{1}$ Peter S. Turner, ${ }^{1}$ \\ and Jonathan C. F. Matthews $\oplus^{1}$ \\ ${ }^{1}$ Quantum Engineering Technologies Labs, H. H. Wills Physics Laboratory and Department of Electrical \& Electronic Engineering, \\ University of Bristol, Bristol BS8 1FD, United Kingdom \\ ${ }^{2}$ Quantum Engineering Centre for Doctoral Training, Nanoscience and Quantum Information Centre, University of Bristol, \\ Bristol BS8 1FD, United Kingdom
}

(Received 10 December 2019; accepted 7 July 2020; published 12 August 2020)

\begin{abstract}
The utility of transmission measurement has made it a target for quantum enhanced measurement strategies. Here we find if the length of an absorbing object is a controllable variable, then, via the Beer-Lambert law, classical strategies can be optimized to reach within $83 \%$ of the absolute quantum limit in precision. Our analysis includes experimental losses, detector noise, and input states with arbitrary photon statistics. We derive optimal operating conditions for both classical and quantum sources, and observe experimental agreement with theory using Fock and thermal states.
\end{abstract}

DOI: 10.1103/PhysRevResearch.2.033243

\section{INTRODUCTION}

In sensing, a measured parameter is often a function of known variables of the apparatus and a variable of interest. An example is the Beer-Lambert law, where the intensity of radiation transmitted through a sample of length $L$ is given by $I=I_{0} \exp (-a L)$, where $I_{0}$ and $I$ are the intensity before and after the sample, and $a$ is the sample absorbance parameter. The law is ubiquitous across investigative techniques, including atomic vapor thermometry [1], femtosecond pumpprobe spectroscopy [2], high-throughput screening [3], onand in-line food processing [4], medical diagnostics [5], and spectrophotometry [6]. The Beer-Lambert law also applies to nonoptical techniques such as neutron transmission and electron tomography $[7,8]$.

Quantum metrology investigates how quantum strategies, such as probing with quantum states, provide increased precision or sensitivity over classical techniques in estimating parameters including optical phase [9,10], transmission [11,12], polarization [13], and displacements [14]. Many practical implementations have demonstrated these schemes, outperforming classical strategies operating at the same average intensity [15-17].

Motivated by the utility of measuring optical transmission to image and identify objects, there have been explorations of the benefits of using quantum light to estimate transmission $\eta$, given by $I=\eta I_{0}[12,18,19]$, including practical demonstrations of twin-beam strategies to provide unbiased

\footnotetext{
*euan.allen@bristol.ac.uk

Published by the American Physical Society under the terms of the Creative Commons Attribution 4.0 International license. Further distribution of this work must maintain attribution to the author(s) and the published article's title, journal citation, and DOI.
}

estimation [20], feed-forward circuits for improved source metrics [11], and microscopy [21,22]. Here, we investigate the advantage of applying optical quantum states for estimation of the absorbance coefficient $a$ (loss per unit length) used in the Beer-Lambert law [23,24]. We compare the output with previous results on estimation of the transmission coefficient $\eta$, which is directly related via $\eta=\exp (-a L)$.

\section{THEORY}

\section{A. Quantum absorbance estimation with loss}

The scenario we consider (Fig. 1) comprises the targeted absorbance $a \in[0, \infty)$, a variable length $L \in(0, \infty)$, lengthindependent loss $\gamma$ which, for example, could arise from loss at each facet of the sample chamber, and length-dependent loss per unit length $\beta$, which we refer to as copropagating loss, and could arise from a distinct absorbing material in the chamber with the sample. Input intensity $I_{0}$ is then related to output $I$ by

$$
I=I_{0} \exp (-a L) \eta_{l},
$$

where we group the instrumental "nonsample" loss mechanisms as $\eta_{l}=\gamma^{2} \exp (-\beta L)$ and facet loss is assumed to be the same for entrance and exit to simplify expressions. Variables $L, \beta$, and $\gamma$ are assumed to be known a priori to infinite precision and so contribute no uncertainty to estimating $a$. In practice, these variables will have uncertainty which will contribute to the uncertainty in $a$, the impact of which can be found using error propagation [25]. We note that both length-dependent loss variables $a$ and $\beta$ are multiplied by the same length $L$ as any loss occurring outside of $L$ can be encapsulated in $\gamma$.

To compare different experimental strategies, we use the Fisher information per average incident photon into the sample, $\mathcal{F}(x)=F(x) / \bar{N}_{0} . F(x)$ is the total Fisher information for the probe state on parameter $x . \bar{N}_{0}$ is the mean input pho- 


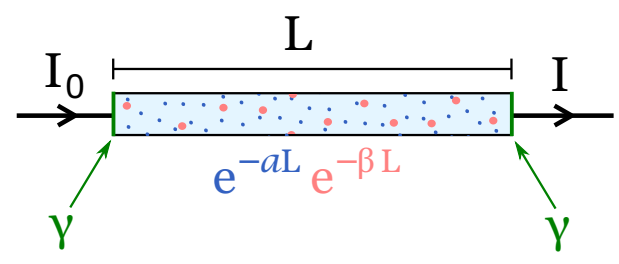

FIG. 1. A typical experiment to measure the absorbance of a medium in the presence of other loss mechanisms such as surface losses $\gamma$ and copropagation loss $\beta$.

ton number. Using Fisher information per absorbed photon, $F /\left[\bar{N}_{0}(1-\eta)\right]$, does not alter the qualitative results observed, but can alter the optimal numerical values of the parameters (which can still be analytically defined).

For estimating transmission $\eta$ (or absorption $1-\eta$ ), a theoretical optimal strategy (per unit intensity) is to input Fock states, $\left|N_{0}\right\rangle$, of known photon number $N_{0}$ into the sample, and perform photon counting at the output $[18,19]$. This single-mode strategy saturates the quantum Cramér-Rao bound for loss estimation and therefore bounds the precision provided by any quantum state of light, including twin-beam strategies [11,12,20] and bright squeezed states [14]. The corresponding classical strategy uses coherent states $|\alpha\rangle$, with mean photon number $\bar{N}_{0}=|\alpha|^{2}$. The underlying evolution of any input state in absorbance and transmission estimation is the same physical process: a loss channel. Therefore, the Fisher information of transmission and absorbance are proportional to one another [Eq. (2)] [25,26], and so share the same optimal quantum strategy.

Because $\eta=\exp (-a L)$ is a continuous differentiable function of $a$, the relationship relating the Fisher information on each parameter applies [25],

$$
\mathcal{F}(a)=\left(\frac{\partial \eta}{\partial a}\right)^{2} \mathcal{F}(\eta)=L^{2} e^{-2 a L} \mathcal{F}(\eta)
$$

For transmission estimation with $\eta_{l}$ present, for classical and quantum strategies, we have $[11,23]$

$$
\begin{gathered}
\mathcal{F}_{C}(\eta)=\frac{\eta_{l}}{\eta}=\eta_{l} e^{a L}, \\
\mathcal{F}_{Q}(\eta)=\frac{\eta_{l}}{\eta\left(1-\eta \eta_{l}\right)}=\frac{\eta_{l} e^{a L}}{1-\eta_{l} e^{-a L}},
\end{gathered}
$$

where $\mathcal{F}_{C}(\eta)$ and $\mathcal{F}_{Q}(\eta)$ define the respective fundamental precision bounds for classical and quantum strategies in estimating $\eta$. When combined with Eq. (2), Eqs. (3) and (4) provide Fisher information per incident photon for estimating $a$,

$$
\begin{aligned}
& \mathcal{F}_{C}(a)=L^{2} \gamma^{2} e^{-(a+\beta) L}, \\
& \mathcal{F}_{Q}(a)=\frac{L^{2} \gamma^{2}}{e^{(a+\beta) L}-\gamma^{2}} .
\end{aligned}
$$

These are plotted in Fig. 2(a) for a fixed $L=1$ and no experimental loss $\eta_{l}=1$, along with the quantum advantage $\mathcal{Q}(a)=\mathcal{F}_{Q}(a) / \mathcal{F}_{C}(a)$. We find fixing $L$ provides a scaling for $\mathcal{Q}(a)$ that follows the trend of transmission estimation: $\mathcal{Q}(a) \rightarrow \infty$ for $a \rightarrow 0[\mathcal{Q}(\eta) \rightarrow \infty$ for $\eta \rightarrow 1]$ [11].

We see in Fig. 2(b) how $\mathcal{F}(a)$ changes for a fixed sample absorbance $(a=1)$, no experimental loss $\eta_{l}=1$, and varying $L$. This differs from Fig. 2(a) and from previous results for absorption estimation that show a degradation of the quantum state Fisher information for increased total loss $[11,12,23]$. For varying length, both $\mathcal{F}_{C}(a)$ and $\mathcal{F}_{Q}(a)$ have maximal values before tending towards zero for both large and small $L$. This demonstrates that for a particular $a$ (e.g., a particular gas fixed in concentration), optimizing the length of the medium that the light passes through provides maximum information on $a$. The optimal $\mathcal{F}_{C}(a)$ can be found by solving $\partial \mathcal{F}_{C}(a) / \partial L=0$,

$$
\begin{aligned}
\frac{\partial \mathcal{F}_{C}(a)}{\partial L} & =2 L \gamma^{2} e^{-(a+\beta) L}-(a+\beta) L^{2} \gamma^{2} e^{-(a+\beta) L} \\
& =[2-(a+\beta) L] L \gamma^{2} e^{-(a+\beta) L}=0,
\end{aligned}
$$

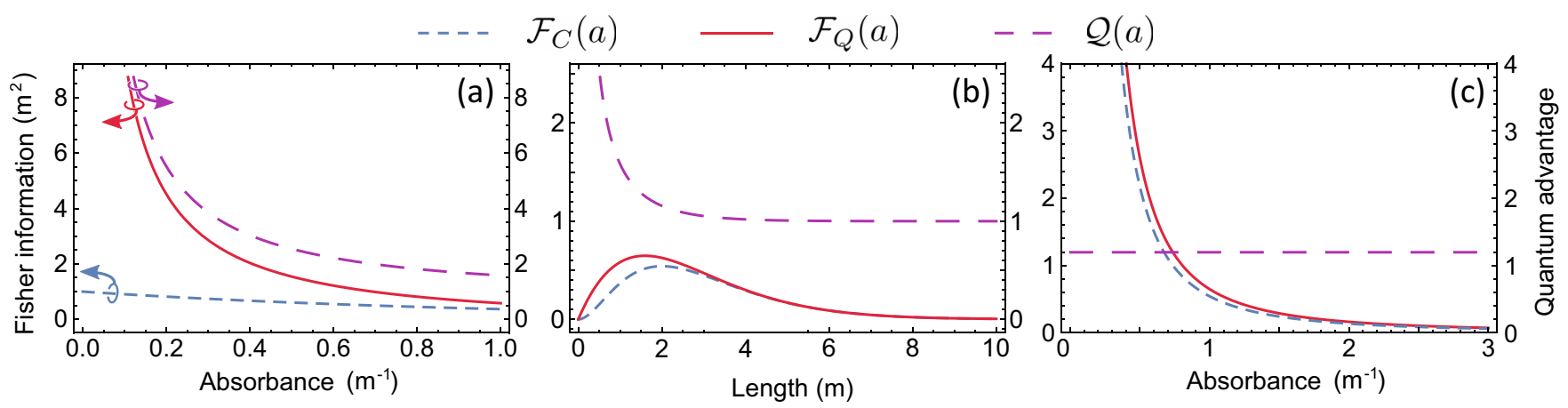

FIG. 2. (a) $\mathcal{F}(a)$ given a fixed $L=1$ for both classical and quantum strategies. Also shown is the quantum advantage $\mathcal{Q}(a)=$ $\mathcal{F}_{C}(a) / \mathcal{F}_{C}(a)$. The trend follows that previously found in transmission estimation [23], where weakly absorbing samples are most improved by using quantum states of light. Color arrows dictate the relevant axis for each line. (b) $\mathcal{F}(a)$ and $\mathcal{Q}(a)$ given a fixed sample absorbance of $a=1$. We see that the Fisher information in both classical and quantum cases peaks at particular length values. (c) $\mathcal{F}(a)$ and $\mathcal{Q}(a)$ when both schemes are allowed to operate at the optimal length values given by Eqs. (8) and (9). We see that maximizing the Fisher information in the classical and quantum case means that the quantum advantage is fixed to a value of 1.2 for all values of absorbance. 

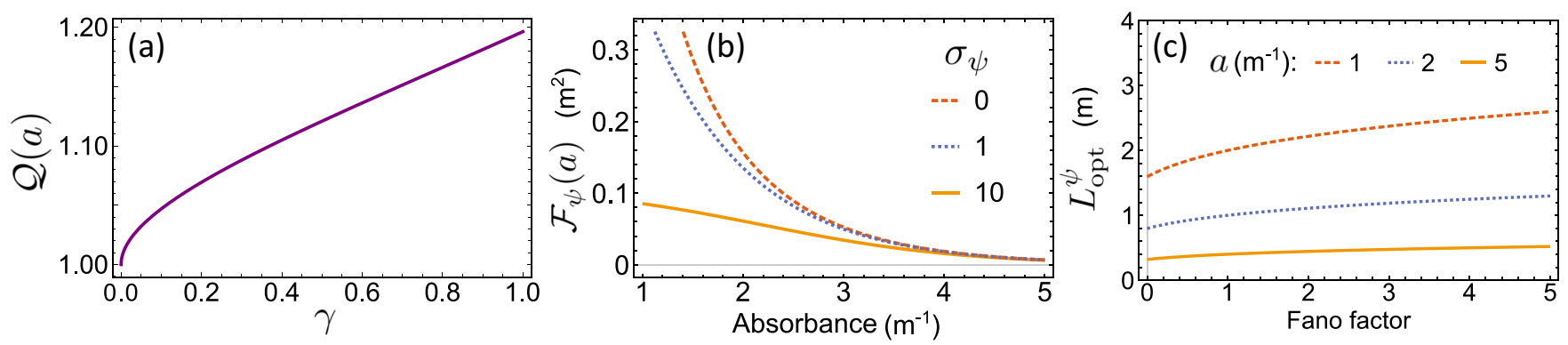

FIG. 3. (a) The effect of facet transmission $\gamma$ on the maximally achievable quantum advantage $\mathcal{Q}(a)$ when both classical and quantum strategies are optimized over the length $L_{\text {opt }}$. (b) The dependence of the Fisher information $\mathcal{F}_{\psi}(a)$ as a function of absorbance $a$ for different input state Fano factors $\sigma_{\psi}$. The sample length is set to $L=1$. (c) The optimal sample length as a function of input state Fano factor for three sample absorbance values.

which is only satisfied by $(a+\beta) L=2$ for nonzero $L$ and $a$. Therefore, for a coherent state,

$$
L_{\mathrm{opt}}^{C}=2 /(a+\beta)
$$

maximizes $\mathcal{F}_{C}(a)$. For $a=1, \beta=0$, and $\gamma=1$, this is in agreement with Fig. 2(b). An identical process for $\mathcal{F}_{Q}(a)$ yields the optimal

$$
L_{\mathrm{opt}}^{Q}=\frac{\mathcal{W}\left[-2 \gamma^{2} / e^{2}\right]+2}{a+\beta},
$$

where $\mathcal{W}[x]$ is the principal value of the Lambert $W$ function [27]. As the values of $L_{\mathrm{opt}}$ are inversely proportional to $a$, the optimal lengths correspond to constant total transmission values. For $\beta=0$ and $\gamma=1$, these are $\eta_{\text {optimal }}^{Q}=$ 0.20 and $\eta_{\text {optimal }}^{C}=0.14$. This shows that for any fixed $a$, $L$ should be chosen for $\gtrsim 80 \%$ total absorption through the sample.

We now compare $\mathcal{F}_{C}(a)$ and $\mathcal{F}_{Q}(a)$ when both schemes are allowed their optimal $L_{\text {opt }}$ value. We compute the quantum advantage as a function of absorbance where at each value of $a$ the length of the material is set to $L_{\mathrm{opt}}(a)$ [Fig. 2(c)]. We find that where there are no constraints on $L$, the advantage gained by using quantum states of light is limited to a fixed factor of $\mathcal{Q}(a)=1.2$, for any $a$. Equivalently, this shows classical light can reach to within $83 \%$ of the fundamental quantum bound. Free choice of the $L$ limits the benefit of implementing a quantum strategy for estimating the absorbance. This can also be seen analytically by inputting the respective values for $L_{\mathrm{opt}}$ into the expression for the quantum advantage $\mathcal{Q}(a)$.

Copropagating loss $\beta$ reduces the Fisher information for both classical and quantum strategies, and in both cases also impacts $L_{\text {opt }}$. However, the Fisher information in the two schemes is reduced by the same factor and therefore the ratio between them, $\mathcal{Q}(a)$, is independent of this type of loss. This is not true for the facet transmission factor $\gamma$, which has a more detrimental effect on the quantum bound than the classical one [Fig. 3(a)].

An important feature of $L_{\mathrm{opt}}$ is that for both strategies, it is a function of absorbance. Since $a$ is the parameter being estimated, there are cases where it will not be known in advance. There do, however, exist practical scenarios when one may still be able to implement $L_{\text {opt }}$. One is when the experiment is intended to measure deviation from an initially known $a$ by $\delta a$, such as a change in the concentration of a gas. Another is when $a$ is known approximately and the quantum strategy is employed to achieve a more precise measurement. Finally, in cases when $a$ is completely unknown but $L$ can be easily varied, one could use Bayesian inference to adaptively update the value of $L$ as estimates of $a$ are attained.

\section{B. Quantum absorbance estimation with arbitrary states}

We now expand our analysis to consider general states $|\psi\rangle$, with arbitrary Fano factor $\sigma_{\psi}=\operatorname{Var}\left(N_{0}\right) / \bar{N}_{0}$, where $\operatorname{Var}\left(N_{0}\right)$ and $\bar{N}_{0}$ are the input photon-number variance and mean, respectively. This allows the estimation capabilities of any light source with any photon statistics to be found. For simplicity, the following analysis assumes that there is no extra experimental loss present: $\eta_{l}=1$.

Let $X\left(N_{0}\right)$ define the (arbitrary) photon-number distribution describing the input state of light used to probe a sample of transmission $\eta$, where $N_{0}$ is the input photon number. As we are interested in linear absorption processes, where the loss acts independently on each incoming photon, the sample applies a Bernoulli trial (binomial distribution) to the input distribution and results in the output compound distribution,

$$
\mathcal{X}_{B}(N \mid \eta)=\sum_{N_{0}=0}^{\infty} X\left(N_{0}\right) B\left(N \mid N_{0}, \eta\right),
$$

where $B\left(N \mid N_{0}, \eta\right)$ defines a binomial distribution providing the probability of measuring $N$ successfully transmitted photons with $N_{0}$ trials and a probability of transmission $\eta$. The expectation values and variance of the binomial distribution,

$$
B\left(N \mid N_{0}, \eta\right)=\left(\begin{array}{c}
N_{0} \\
N
\end{array}\right) \eta^{N}(1-\eta)^{N_{0}-N},
$$

can be calculated to be

$$
\begin{gathered}
\mathbb{E}_{B}[N]=N_{0} \eta, \\
\mathbb{E}_{B}\left[N^{2}\right]=\eta^{2} N_{0}^{2}+\eta(1-\eta) N_{0}, \\
\operatorname{Var}_{B}(N)=N_{0} \eta(1-\eta),
\end{gathered}
$$


where $\mathbb{E}_{F}[x]$ denotes the expectation value of $x$ for the distribution $F$. For the compound distribution $\mathcal{X}_{B}(N \mid \eta)$,

$$
\begin{aligned}
\mathbb{E}_{\mathcal{X}_{B}}[N] & =\sum_{N=0}^{\infty} N \mathcal{X}_{B}(N \mid \eta) \\
& =\sum_{N=0}^{\infty} N \sum_{N_{0}=0}^{\infty} X\left(N_{0}\right) B\left(N \mid N_{0}, \eta\right) \\
& =\sum_{N_{0}=0}^{\infty} X\left(N_{0}\right) \mathbb{E}_{B}[N] \\
& =\sum_{N_{0}=0}^{\infty} X\left(N_{0}\right) N_{0} \eta=\eta \mathbb{E}_{X}\left[N_{0}\right], \\
\mathbb{E}_{\mathcal{X}_{B}}\left[N^{2}\right] & =\sum_{N=0}^{\infty} N^{2} \sum_{N_{0}=0}^{\infty} X\left(N_{0}\right) B\left(N \mid N_{0}, \eta\right) \\
& =\sum_{N_{0}=0}^{\infty} X\left(N_{0}\right)\left[N_{0}^{2} \eta^{2}+N_{0}\left(\eta-\eta^{2}\right)\right] \\
& =\eta^{2} \mathbb{E}_{X}\left[N_{0}^{2}\right]+\eta(1-\eta) \mathbb{E}_{X}\left[N_{0}\right], \\
\operatorname{Var}_{\mathcal{X}_{B}}(N)= & \eta^{2} \operatorname{Var}_{X}\left(N_{0}\right)+\eta(1-\eta) \mathbb{E}_{X}\left[N_{0}\right] .
\end{aligned}
$$

From Eq. (17), we see that for an input photon-number distribution of $X\left(N_{0}\right)$, the photon-number variance of the light after the sample (where the sample transmission coefficient is $\eta)$ is given by

$$
\operatorname{Var}_{\mathcal{X}_{B}}(N)=\eta^{2} \operatorname{Var}\left(N_{0}\right)+(1-\eta) \eta \bar{N}_{0},
$$

where $\mathbb{E}_{X}\left[N_{0}\right]=\bar{N}_{0}$ is the mean number of input photons and $\operatorname{Var}\left(N_{0}\right)$ is the variance. Using the estimator $\hat{\eta}=N / \bar{N}_{0}$ allows the variance on the estimator to be computed using the propagation of errors, $\operatorname{Var}(\hat{\eta})=\left(\frac{\partial \hat{\eta}}{\partial N}\right)^{2} \operatorname{Var}_{\mathcal{X}_{B}}(N)$ :

$$
\begin{aligned}
\operatorname{Var}(\hat{\eta}) & =\frac{\operatorname{Var}(N)_{\mathcal{X}_{B}}}{\bar{N}_{0}^{2}} \\
& =\frac{1}{\bar{N}_{0}}\left[\eta^{2} \sigma_{\psi}+(1-\eta) \eta\right],
\end{aligned}
$$

where $\sigma_{\psi}=\operatorname{Var}\left(N_{0}\right) / \bar{N}_{0}$ is the Fano factor of the input state. From this, we find that the Fisher information on the parameter $\eta$ per incident photon is given by

$$
\mathcal{F}_{\psi}(\eta)=\frac{1}{\eta^{2} \sigma_{\psi}+(1-\eta) \eta} .
$$

Note that the Fisher information is maximized for the case where $\sigma_{\psi}=0$, which is true for Fock states and in agreement with previous results $[18,19]$. The calculated $\mathcal{F}_{\psi}(\eta)$ for Fock and coherent $\left(\sigma_{\psi}=1\right)$ states are also in agreement with previous bounds found in the literature $[11,23]$.

Following Eq. (2), where $\mathcal{F}(\eta)$ is related to $\mathcal{F}(a)$, the Fisher information on $a$ for an arbitrary input state is found to be

$$
\mathcal{F}_{\psi}(a)=L^{2} /\left[\sigma_{\psi}+\exp (a L)-1\right] .
$$

For coherent and Fock states ( $\sigma_{\psi}=1$ and 0 , respectively), $\mathcal{F}_{\psi}(a)$ returns to $\mathcal{F}_{C}(a)$ and $\mathcal{F}_{Q}(a)$. Equation (21) can com-

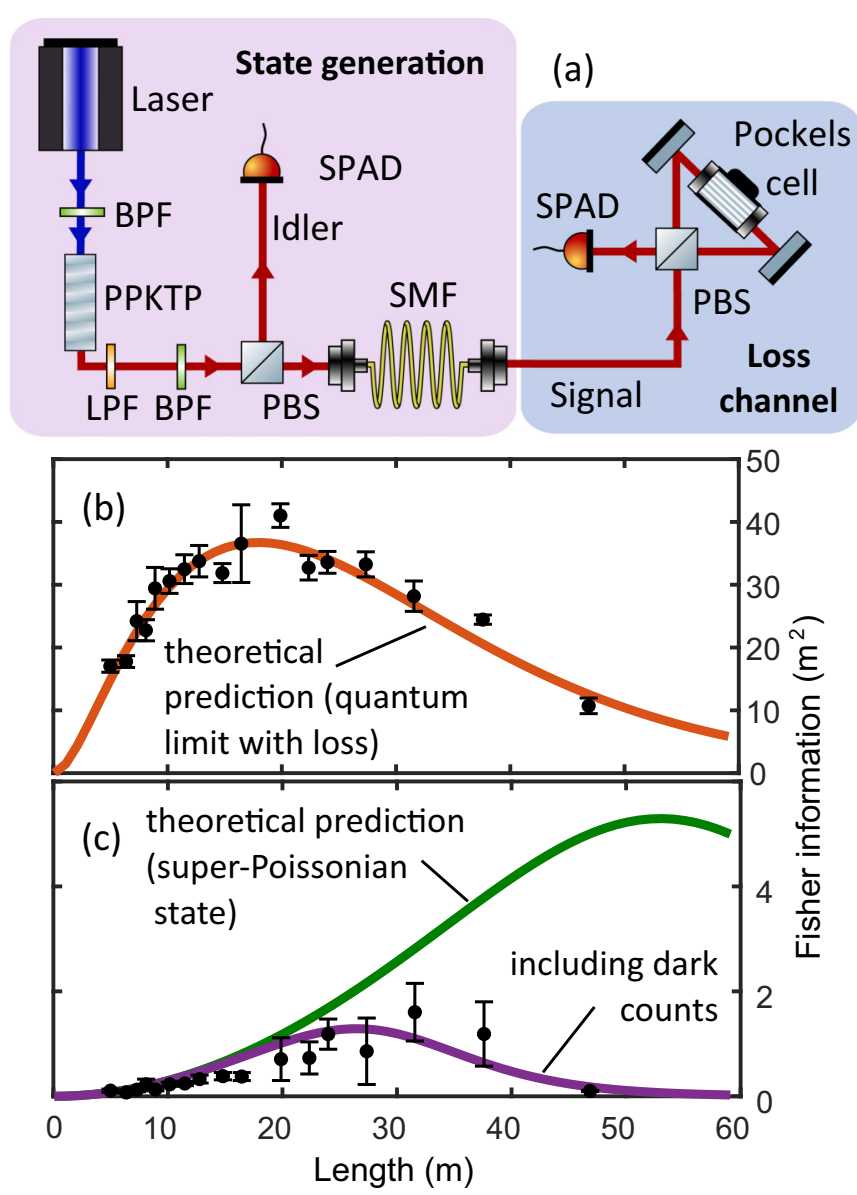

FIG. 4. (a) Experimental setup to measure optimization of sample length. (b) Results for pair-photon source (c) Results for singlearmed source with and without dark counts included in the theoretical analysis.

pute the estimation capabilities of any quantum probe state, including displaced (bright) squeezed states [28].

The dependence of the optimal length on $\sigma_{\psi}$ is found analytically to be

$$
L_{\mathrm{opt}}^{\psi}=\left\{\mathcal{W}\left[2\left(\sigma_{\psi}-1\right) / e^{2}\right]+2\right\} / a .
$$

The dependence of $\mathcal{F}_{\psi}(a)$ and $L_{\mathrm{opt}}^{\psi}$ on the Fano factor and absorbance $a$ are shown in Figs. 3(b) and 3(c), respectively.

The general features of absorbance estimation also appear in multipass strategies for quantum and classical light. Specifically, the freedom of multiple passes acts like a discrete version of optimizing $L$ and allows $\mathcal{F}_{C}$ to approach the value of $\mathcal{F}_{Q}$. This has been demonstrated by Birchall et al. who investigated multipass strategies in lossy phase estimation [29] and loss estimation [30].

\section{EXPERIMENT}

We experimentally demonstrate length-dependent optimization performed for both quantum (single-photon) and noisy (thermal) sources of light using the apparatus in Fig. 4(a) and Ref. [11]. A collinear type-II spontaneous parametric down-conversion source [periodically poled potassium titanyl phosphate (PPKTP) crystal] is pumped by a $3 \mathrm{~mW}$, 
$404 \mathrm{~nm}$ continuous-wave laser to spontaneously produce correlated photon pairs at $810 \mathrm{~nm}$ with orthogonal polarizations. The photon pairs are spatially separated at a polarizing beam splitter (PBS) into "signal" and "idler" channels. The signal photon is detected by a single-photon avalanche photodiode (SPAD) to herald the presence of a single-photon Fock state (idler). Long-pass (LPF) and band-pass filters (BPF) exclude the pump.

The sample of varying loss is a Pockels cell modulator. When used in conjunction with a PBS Sagnac, this applies a variable loss to the incoming photon independent of polarization [11]. The transmission of the idler photon's path with the switch fully open is $38 \%$, including detection efficiency, and thus we apply $\gamma=\sqrt{0.38}=0.62$ and $\beta=0$ to the following analysis. The experiment produced approximately 14 $\mathrm{k}$ coincidences $/ \mathrm{s}$.

Optimization of $L$ is observed by applying the inferred total loss from an object with absorbance $a=1 \mathrm{~m}^{-1}$ with varying $L$. For example, for $a=1 \mathrm{~m}^{-1}, L=\{1,3,5\} \mathrm{m}$ applies losses $\eta=\exp (-a L)=\{0.37,0.14,0.05\}$, which we implement with the Pockels cell. By setting each $\eta$ and then taking measurements to estimate $a$ (given that we have prior knowledge of $L$ ), we measure the statistics of the noise on the estimate of absorbance and to estimate $\mathcal{F}(a)=1 / \operatorname{Var}(\hat{a})$, where $\operatorname{Var}(\hat{a})$ denotes the variance on the estimates of the parameter $a$.

Estimates of $a$ are found by using

$$
\hat{a}=-\ln \left(N_{c c} / \gamma N_{S}\right) / L,
$$

where $N_{c c}$ is the number of coincidences between signal and idler SPADs, and $N_{S}$ is the total number in the signal channel [11]. Equation (23) is only valid for the Fisher information per incident photon metric where instrumental loss occurring before and after the sample affect $\mathcal{F}$ in the same fashion, and therefore do not need to be considered independently. A total of 500 estimates for each setting of absorbance were found using a coincidence window of 0.5 seconds. These were separated into five groups of 100 estimates, and the variance of each group computed, each providing a Fisher information estimate. Their means are shown in Fig. 4(b), with error bars computed using the standard error of the Fisher information estimates. These show good agreement with theoretical predictions for the optimal quantum strategy.

\section{A. Single arm estimation and the effects of dark counts}

By disregarding the idler photons, we experimentally test the estimation capabilities of a noisy (super-Poissonian) light source as a single arm of a pair-photon source is a thermal state [31]. Here, the estimator for the absorbance is changed to

$$
\hat{a}=-\ln \left[\left(N_{I}-\bar{N}_{\mathrm{DC}}\right) / \bar{N}_{0}\right] / L,
$$

where $N_{I}$ is the number of idler photons detected, $\bar{N}_{\text {DC }}$ is the mean number of dark counts in the idler detector, and $\bar{N}_{0}$ is the mean number of input photons, found prior by applying no loss.

The results in Fig. 4(c) deviate from the theory given by Eq. (20). We attribute this to dark counts from the SPADs, which are particularly detrimental for the thermal state (single-arm) strategy as the counts from the lossy arm are used to estimate $a$. This is in contrast to the more robust coincidence estimator [Eq. (23)] where only the singles in the signal channel are considered. By adding dark counts into the analysis, we are able to reconcile the difference between theoretical predictions and the experimental results seen in Fig. 4(c).

For a single-arm measurement, estimates of $\eta$ are calculated using the estimator

$$
\hat{\eta}=\frac{N_{I}-\bar{N}_{\mathrm{DC}}}{\bar{N}_{0}},
$$

where $N_{I}$ is number of detector counts in a sample window, $\bar{N}_{\text {DC }}$ is the average number of dark counts over many sample windows, and $\bar{N}_{0}$ is the average number of input photons. The number of counts on the detector is the sum of dark counts in the sample window and the number of incident photons, $N_{i}, N_{I}=N_{\text {DC }}+N_{i}$. As $N_{i}$ and $N_{\text {DC }}$ are independent variables, the variance on $N_{I}$ is the sum of the individual variances of $N_{i}$ and $N_{\text {DC }}$,

$$
\operatorname{Var}\left(N_{I}\right)=\operatorname{Var}\left(N_{i}\right)+\operatorname{Var}\left(N_{\mathrm{DC}}\right) .
$$

Using error propagation, $\operatorname{Var}(\hat{\eta})=\left(\frac{\partial \hat{\eta}}{\partial N_{I}}\right)^{2} \operatorname{Var}\left(N_{I}\right)$, and Eq. (25), we find

$$
\operatorname{Var}(\hat{\eta})=\frac{\operatorname{Var}\left(N_{I}\right)}{\bar{N}_{0}^{2}}=\frac{\operatorname{Var}\left(N_{i}\right)+\operatorname{Var}\left(N_{\mathrm{DC}}\right)}{\bar{N}_{0}^{2}} .
$$

We can relate the detected incident photon-number variance with the input (presample) variance using Eq. (18),

$$
\operatorname{Var}\left(N_{i}\right)=\eta^{2} \operatorname{Var}\left(N_{0}\right)+(1-\eta) \eta \bar{N}_{0},
$$

which can then be used to give the variance on $\hat{\eta}$ for an arbitrary input state with Fano factor $\sigma_{\psi}=\operatorname{Var}\left(N_{0}\right) / \bar{N}_{0}$,

$$
\operatorname{Var}(\hat{\eta})=\frac{1}{\bar{N}_{0}}\left[\frac{\operatorname{Var}\left(N_{\mathrm{DC}}\right)}{\bar{N}_{0}}+\eta^{2} \sigma_{\psi}+(1-\eta) \eta\right]
$$

This provides an estimator information per incident photon of

$$
I(\eta)=\frac{1}{\operatorname{Var}\left(N_{\mathrm{DC}}\right) / \bar{N}_{0}+\eta^{2} \sigma_{\psi}+(1-\eta) \eta},
$$

which simplifies to the Fisher information bounds for previously discussed scenarios $\left[\operatorname{Var}\left(N_{\mathrm{DC}}\right)=0\right]$. This can be related to the information on the absorbance $a$ by using Eq. (2) and produces the corrected theoretical line in Fig. 4(c).

We estimate the $\sigma_{\psi}$ value for the idler path by measuring the photon-number variance and mean when there is no sample present and correcting for the inherent loss of the channel. By rearranging Eq. (18), we find

$$
\sigma_{\psi}=\frac{\sigma+\eta_{l}-1}{\eta_{l}}
$$

where $\sigma_{\psi}$ is the Fano factor at the input to a lossy channel with transmission $\eta_{l}$ and $\sigma$ is the Fano factor measured after the channel. Experimentally, the postloss Fano factor was measured to be $\sigma=314.5$ with $\eta_{l}=0.38$. This gave a predicted $\sigma_{\psi}=826$. The variance of detector dark counts $\operatorname{Var}\left(N_{\mathrm{DC}}\right)$ was measured to be 518 . 


\section{CONCLUSION}

We have shown how experimental optimization of the sample length $L$ can offer significant advantages in precision for both quantum and classical inputs in absorbance estimation. We find that for cases where this optimization is possible, the quantum advantage is restricted to 1.2 , at most. We have derived optimal conditions for a number of experimental variations including additional experimental loss, input states with arbitrary photon-number statistics, and detector dark counts. The experimental implementation presented here demonstrates that $L$ can be optimized for Fock and thermal states, suggesting that for such an experiment, quantum advantage is limited. These results not only have implications for future quantum sensors designed for measuring absorbance, but can also be applied to optimize classical sensors using any light source, impacting a range of application areas [1-6].

Fock states are unlikely to find widespread use for high-precision measurements because the experiments that operate at the quantum limit in precision per-photon flux use coincidence-based photon-counting measurements [11], which currently limit optical power in the probe to $\sim$ femtowatts. In many cases, the performance of Fock states can be readily surpassed by using a classical source of much higher brightness, albeit at lower precision per photon flux. However, as Fock states saturate the quantum Cramer-Rao bound for loss estimation, they bound the ultimate precision achievable by any state for any fixed photon number. Our work outlines a strategy of experimental optimization of the much more practical coherent state that reaches $83 \%$ of the precision enhancement defined by this ultimate quantum limit. Shot-noise limited intensity measurements are sufficient for this strategy, opening the way for a practical dynamic range of probe intensity even in cases using a noisy laser source [32].

The existence of experimental optimization strategies for absorbance, phase [29], and loss estimation [30] suggests that in general, the existence of a free optimization parameter can be a powerful tool for increasing the achievable measurement precision. Future analysis and experiments should consider this to correctly predict the advantages of quantum strategies. Efforts towards providing practical advantages using quantum light can now be focused towards applications where such optimization strategies are difficult to implement, such as imaging or very weakly absorbing samples.

Data are available at the University of Bristol data repository [33].

\section{ACKNOWLEDGMENTS}

We thank P. Birchall, J. Mueller, and S. Wollmann for helpful discussions and feedback on the manuscript. This work was supported by the Centre for Nanoscience and Quantum Information (NSQI) and the Engineering and Physical Sciences Research Council (Grants No. EP/M01326X/1, No. EP/T00097X/1, and No. EP/R024170/1). E.J.A. was supported by the Quantum Engineering Centre for Doctoral Training, EPSRC Grant No. EP/L015730/1 and EPSRC Doctoral Prize Fellowship No. EP/R513179/1. J.C.F.M. acknowledges support from ERC Grant No. ERC-2018- STG803665.
[1] G.-W. Truong, E. F. May, T. M. Stace, and A. N. Luiten, Phys. Rev. A 83, 033805 (2011).

[2] S. Woutersen, U. Emmerichs, and H. J. Bakker, Science 278, 658 (1997).

[3] J. Inglese et al., Nat. Chem. Biol. 3, 466 (2007).

[4] C. Scotter, Food Control 1, 142 (1990).

[5] S. Behera, S. Ghanty, F. Ahmad, S. Santra, and S. Banerjee, J. Anal. Bioanal. Techn. 3, 151 (2012).

[6] T. A. Trumbo, E. Schultz, M. G. Borland, and M. E. Pugh, Biochem. Mol. Biol. Educ. 41, 242 (2013).

[7] P. Vontobel, E. H. Lehmann, R. Hassanein, and G. Frei, Physica B: Condens. Matter 385-386, 475 (2006).

[8] R. Yan, T. J. Edwards, L. M. Pankratz, R. J. Kuhn, J. K. Lanman, J. Liu, and W. Jiang, J. Struct. Biol. 192, 297 (2015).

[9] C. M. Caves, Phys. Rev. D 23, 1693 (1981).

[10] T. Ono, R. Okamoto, and S. Takeuchi, Nat. Commun. 4, 2426 (2013).

[11] J. Sabines-Chesterking et al., Phys. Rev. Appl. 8, 014016 (2017).

[12] P.-A. Moreau et al., Sci. Rep. 7, 6256 (2017).

[13] L. Zhang, K. W. C. Chan, and P. K. Verma, Phys. Rev. A 93, 032137 (2016).

[14] M. Xiao, L.-A. Wu, and H. J. Kimble, Opt. Lett. 13, 476 (1988).

[15] M. A. Taylor and W. P. Bowen, Phys. Rep. 615, 1 (2016).
[16] V. Giovannetti, S. Lloyd, and L. Maccone, Nat. Photon. 5, 222 (2011).

[17] R. Schnabel, N. Mavalvala, D. E. McClelland, and P. K. Lam, Nat. Commun. 1, 121 (2010).

[18] A. Fujiwara, Phys. Rev. A 70, 012317 (2004).

[19] G. Adesso, F. Dell'Anno, S. De Siena, F. Illuminati, and L. A. M. Souza, Phys. Rev. A 79, 040305 (2009).

[20] E. Losero, I. Ruo-Berchera, A. Meda, A. Avella, and M. Genovese, Sci. Rep. 8, 7431 (2018).

[21] G. Brida, M. Genovese, and I. R. Berchera, Nat. Photon. 4, 227 (2010).

[22] J. Sabines-Chesterking, A. McMillan, P. Moreau, S. Joshi, S. Knauer, E. Johnston, J. Rarity, and J. C. F. Matthews, Opt. Express 27, 30810 (2019).

[23] R. Whittaker et al., New J. Phys. 19, 023013 (2017).

[24] G. Spedalieri, S. Pirandola, and S. L. Braunstein, Phys. Rev. A 98, 053836 (2018).

[25] E. L. Lehmann and G. Casella, Theory of Point Estimation (Springer Science \& Business Media, New York, 2006).

[26] M. G. A. Paris, Int. J. Quantum Inf. 7, 125 (2009).

[27] R. M. Corless, G. H. Gonnet, D. E. G. Hare, D. J. Jeffrey, and D. E. Knuth, Adv. Comput. Math. 5, 329 (1996).

[28] The Fano factor for a displaced squeezed state is given by $\sigma=\left(|\alpha|^{2} e^{-2 r}+2 \sinh ^{2} r \cosh ^{2} r\right) /\left(\sinh ^{2} r+|\alpha|^{2}\right)$, where $\alpha$ is the coherent amplitude and $r$ is the squeezing parameter. 
[29] P. M. Birchall, J. L. O'Brien, J. C. F. Matthews, and H. Cable, Phys. Rev. A 96, 062109 (2017).

[30] P. M. Birchall, E. J. Allen, T. M. Stace, J. L. O'Brien, J. C. F. Matthews, and H. Cable, Phys. Rev. Lett. 124, 140501 (2020).

[31] B. Blauensteiner, I. Herbauts, S. Bettelli, A. Poppe, and H. Hübel, Phys. Rev. A 79, 063846 (2009).
[32] E. J. Allen, G. Ferranti, K. R. Rusimova, R. J. A. Francis-Jones, M. Azini, D. H. Mahler, T. C. Ralph, P. J. Mosley, and J. C. F. Matthews, Phys. Rev. Appl. 12, 044073 (2019).

[33] E. Allen, Data from "Approaching the Quantum Limit of Precision in Absorbance Estimation Using Classical Resources," https://doi.org/10.5523/bris.3e22hupanhca522bibwhfemzp7 (2020). 